\title{
Nonlinear Analysis of Smart Concrete Beams with Piezoelectric Actuators
}

\author{
Weichen Xue ${ }^{1, a}$, Yang $\mathrm{Li}^{1, \mathrm{~b}}$ and Feng Yang ${ }^{1, \mathrm{c}}$ \\ ${ }^{1}$ Department of Structural Engineering, College of Civil Engineering, Tongji University, Shanghai \\ 200092, China \\ axuewc@tongji.edu.cn, 026_liyang@tongji.edu.cn, cyangfeng_tj@163.com
}

\begin{abstract}
Keywords: Smart concrete beam, piezoelectric actuator, active control, nonlinear analysis, parametric analysis, recovery ratio

Abstract. A novel piezoelectric smart concrete beam composed of a concrete beam, a piezoelectric actuator and a counterforce device is developed and investigated. Based on the governing equation of 'actuating force-voltage' of piezoelectric actuator proposed by the authors, a nonlinear analysis program, in which material nonlinearity, geometric nonlinearity and local contact effects of cracked section are considered, is developed using Fortran language to analyze the piezoelectric smart concrete beams under active deflection control. Then active control tests are conducted to verify the developed program and good agreement is obtained. Moreover, a parametric analysis is carried out to investigate the influence of eccentricity of the actuator and the initial damage of concrete beam. The results reveal that piezoelectric actuators have greater active control effect when the eccentricity is larger while the recovery ratio is smaller when the initial damage of concrete beam is more severe.
\end{abstract}

\section{Introduction}

As is well-known, piezoelectric materials have the ability to perform both sensing and actuating functions due to the piezoelectric effects, which enable the materials to transform the electrical energy to mechanical energy or vice versa $[1,2]$. Piezoelectric ceramics, known as low-cost, lightweight and easy-to-implement materials for active control of smart structures, are the most popular and developed amongst the piezoelectric materials [3]. To perform the actuating function, piezoelectric ceramics are available in various forms such as stack type actuators and patch actuators. Compared to patch actuators, stack type piezoelectric actuators can offer many advantages, such as higher output force [4]. Besides, stack type actuators can be incorporated into the structures, with slight modifications and without significantly changing the structural stiffness of the system, and therefore they are the commonly used actuators in civil applications [5]. Though the application of piezoelectric actuators appears firstly in the aerospace industry, with the rapid development of piezoelectric materials, applications of piezoelectric actuators have gradually extended to the field of civil engineering since the beginning of 1990s.

By far, there have been many experimental and theoretical studies on actively controlled smart structures using piezoelectric actuators in civil engineering. However, most of the studies are focused on the active vibration control of smart structures [6, 7]. For example, Nkikkhoo presented the numerical analysis of active vibration control of piezoelectric smart beams and slabs [8,9]. Kamada et al. carried out the active vibration control tests on frame structures [10,11]. However, little research has been done on the investigations of the active deflection control of concrete beam using piezoelectric actuator. As regard to civil structure, the deflection of concrete beam is closely allied to the normal use of structures. An excessive deflection not only presents an unpleasant appearance, but also seriously affects the function and safety of structures. Therefore, it is of great significance to actively control the deflection of concrete beams.

In this paper, a nonlinear analysis program is proposed using Fortran language to analyze the piezoelectric smart concrete beams under active deflection control. In order to verify the developed program, active control tests of piezoelectric smart concrete beams are carried out. Finally, a parametric study is conducted to investigate the impact of eccentricity of the actuator and the initial damage of concrete beam on the active deflection control of piezoelectric smart concrete beams. 


\section{Introduction of piezoelectric smart concrete beam}

The stack type piezoelectric actuator used in the study is provided by German Piezomechanik Gmbn Company. The type of the piezoelectric actuator is PST 150/20/18 VS25, which is assembled with piezoceramic laminar wafers in a cylindrical stainless steel housing. The diameter of main body is $25 \mathrm{~mm}$, and the height is $36 \mathrm{~mm}$. The output end has a height of $4 \mathrm{~mm}$, an outer ring diameter of $10 \mathrm{~mm}$ and an inner ring diameter of $6 \mathrm{~mm}$ (shown in Fig. 1). The internal piezoceramic wafers have a capacitance of $11 \mathrm{~F}$ and a voltage range of $-30 \mathrm{~V}$ to $+150 \mathrm{~V}$.

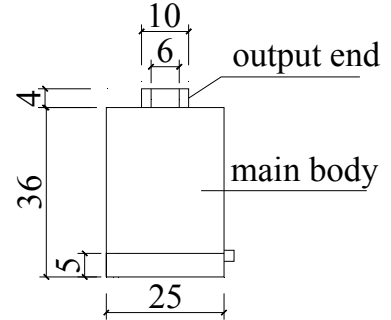

Fig. 1. Dimensions of piezoelectric actuator $[\mathrm{mm}]$

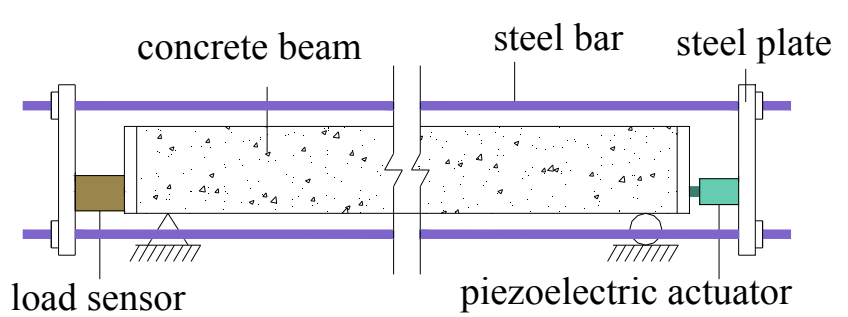

Fig. 2. Configuration of piezoelectric smart concrete beam

A new type of piezoelectric smart concrete beam which consists of a concrete beam as substrate with a piezoelectric actuator eccentrically located at the beam end and a counterforce device which enables the actuating force to act on the beam, is proposed in this paper (shown in Fig. 2). By supplying power to the stack type piezoelectric actuator, an eccentric actuating force will be easily applied at the beam end and therefore the active deflection control of the proposed piezoelectric smart concrete beam can be achieved.

\section{Nonlinear analysis and experimental verification}

Introduction of the program. In previous research, the authors developed a nonlinear analysis program which is capable for the full-range analysis of both reinforced concrete beams and prestressed concrete beams [12]. Actuating force tests of stack type piezoelectric actuator were carried out and the governing equation of 'actuating force-voltage' was established by data fitting, which is expressed as: $F_{V}=3.65 \times V-25.1$, where $F_{V}$ is the output force of piezoelectric actuator and $V$ the input voltage with range of 0 to $150 \mathrm{~V}$ [12]. In this paper, by introducing the governing equation of 'actuating force-voltage' into the developed program, the nonlinear analysis of piezoelectric smart concrete beams under active deflection control can also be well achieved. The main features of the developed program are as follows:

1. For the sake of calculation accuracy and to take the material nonlinearity and geometric nonlinearity into consideration, the element is divided into a finite number of discrete segments along the length. For each segment, the cross section at the midpoint is selected for analysis which is then separated into several layers according to the materials of concrete, steel bars or prestressing tendons.

2 . The M-N- $\phi$ relations are formed directly from physical properties of materials and the element stiffness matrix is derived based on the nonlinear finite element theory and the principle of virtual work, which is composed of three parts that inflect the influences of the material nonlinearity, the geometric nonlinearity as well as the axial second-order moment, respectively.

3. The load increment matrix is deduced using incremental loading method with consideration of the nonlinear characteristics of structures and the loading scheme in the program design is in accordance with the active control tests.

Experiment work. In order to validate the proposed nonlinear analysis program, active control tests were conducted based on four piezoelectric smart concrete beams. The beams were tested under two-point loading. The specimens were made from micro-concrete with small diameter steel wires as reinforcing bars. Besides, all specimens were designed in identical cross sections with width of $40 \mathrm{~mm}$ and depth of $60 \mathrm{~mm}$. Following design parameters are considered: longitudinal reinforcement ratios, span-depth ratios and the eccentricity of actuators. Design details of specimens are summarized in Table 1. 
All specimens experienced two stages during the tests, including the external loading stage and actuating stage. Comparing the mid-span deflection before and after actuating, it was found that obvious inverted arches were observed in all beams and the recovery ratio of deflection of four beams ranged from $20 \%$ to $40 \%$, both of which indicated the active control of deflection was well achieved.

Table 1. Design details of specimens $[\mathrm{mm}]$

\begin{tabular}{lcccc}
\hline Specimens & ZN-1 & ZN-2 & ZN-3 & ZN-4 \\
\hline Compressive reinforcements & $2 ø 1.5$ & $2 ø 1.5$ & $2 ø 2.5$ & $2 ø 2.5$ \\
Tensile reinforcements & $2 ø 1.5$ & $2 ø 1.5$ & $2 ø 3.4$ & $2 ø 3.4$ \\
Reinforcement ratio $\rho$ & $0.16 \%$ & $0.16 \%$ & $0.82 \%$ & $0.82 \%$ \\
Stirrup spacing & $1.5 @ 30$ & $1.5 @ 30$ & $2.5 @ 30$ & $2.5 @ 30$ \\
Eccentricity of actuator & 20 & 20 & 20 & 10 \\
Span-depth ratio & $8.5: 1$ & $7: 1$ & $7: 1$ & $7: 1$ \\
\hline
\end{tabular}

Comparison with experimental data. Nonlinear analysis of four smart concrete beams with piezoelectric actuators is achieved based on the developed program. The numerical results of the proposed program are compared against the experimental data obtained by active control tests. Fig. 3 shows the numerical-experimental comparison of the mid-span deflection. It can be found that good agreement between experimental and numerical results is achieved.
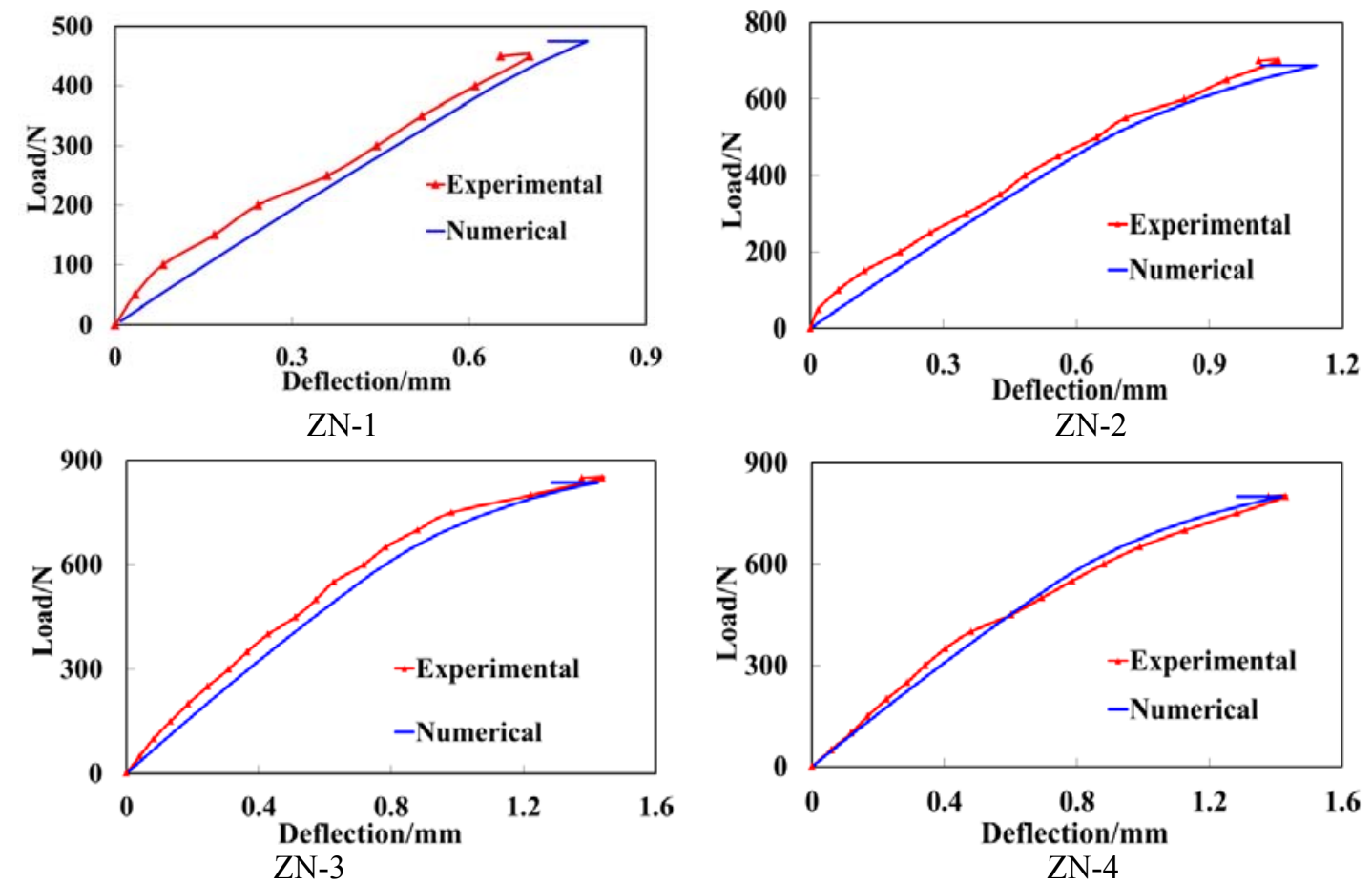

Fig. 3. Numerical-experimental comparison of load-deflection curves for beam ZN-1 ZN-4

From Fig. 3, the following conclusions can be drawn:

1. The deflections of all beams recover to some extent after actuating, which shows a good capacity of deflection recovery of piezoelectric smart concrete beams.

2. At external loading stage, the program predicts the load-deflection curve quite well, indicating that the proposed program can precisely simulate the loading process. While at actuating stage, good agreement is also achieved between numerical and experimental results, which demonstrates that the proposed nonlinear analysis program has a fairly good accuracy.

It is worth noting that the output force produced by the piezoelectric actuator used in this study is limited which leads to the relatively low degree of deflection recovery of smart beams. Once the output force is enlarged, the degree of deflection recovery will be well improved. 


\section{Parametric analysis}

In this section, the proposed program which has been successfully validated above is used to conduct a parametric study to investigate the effects of eccentricity of the actuator and the initial damage of concrete beam on the active deflection control performances. Eight piezoelectric smart concrete beams with engineering size are designed and are divided into two groups (SL-1 SL-4 and SL-5 SL-8). The primary design parameters include crack width and eccentricity of the actuators. The geometrical characteristics of specimens are shown in Table 2. In view of the actual dimensions of structures, the actuating force of piezoelectric actuator is amplified according to the geometric similarity ratio in the program computation.

Table 2. Geometrical characteristics of specimens

\begin{tabular}{lcccccccc}
\hline Specimens & SL-1 & SL-2 & SL-3 & SL-4 & SL-5 & SL-6 & SL-7 & SL-8 \\
\hline Reinforcement ratio $\rho$ & $2.09 \%$ & $2.09 \%$ & $2.09 \%$ & $2.09 \%$ & $0.87 \%$ & $0.87 \%$ & $0.87 \%$ & $0.87 \%$ \\
Span & 1800 & 1800 & 1800 & 1800 & 4200 & 4200 & 4200 & 4200 \\
Span-depth ratio & $9: 1$ & $9: 1$ & $9: 1$ & $9: 1$ & $14: 1$ & $14: 1$ & $14: 1$ & $14: 1$ \\
Section dimension b $\times \mathrm{h}$ & \multicolumn{7}{c}{$100 \times 200$} & \multicolumn{5}{c}{$200 \times 300$} \\
Eccentricities of Actuator & $\mathrm{h} / 4$ & $3 \mathrm{~h} / 8$ & $\mathrm{~h} / 4$ & $3 \mathrm{~h} / 8$ & $\mathrm{~h} / 4$ & $3 \mathrm{~h} / 8$ & $\mathrm{~h} / 4$ & $3 \mathrm{~h} / 8$ \\
Crack width & 0.1 & 0.1 & 0.2 & 0.2 & 0.1 & 0.1 & 0.2 & 0.2 \\
\hline
\end{tabular}

The calculated load-deflection curves of the first group specimens (SL-1 SL-4) and the second group specimens (SL-5 SL-6) are shown in Fig. 4 and Fig 5, respectively.
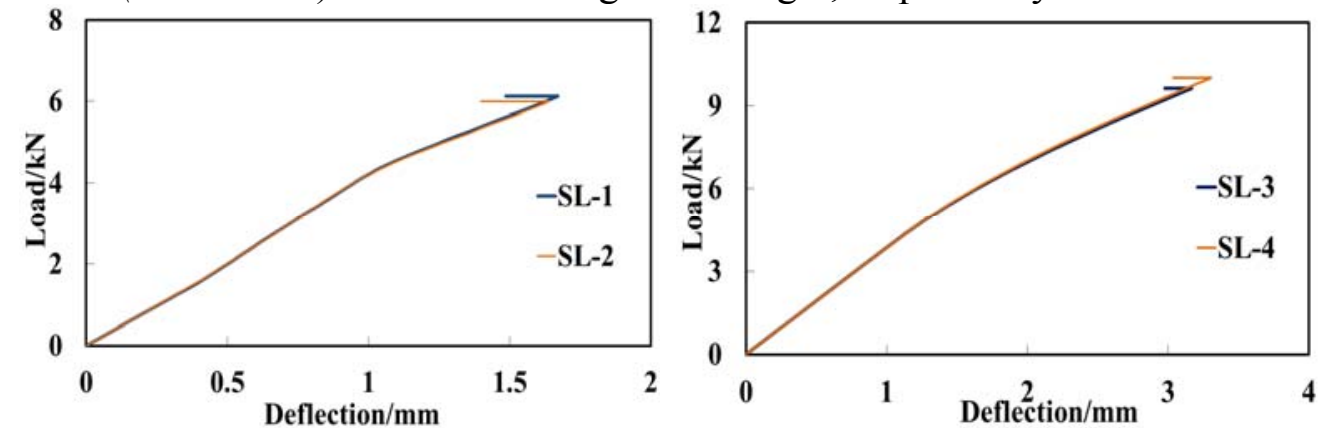

Fig. 4. Curves of load-deflection of first group specimens (SL-1 SL-4)
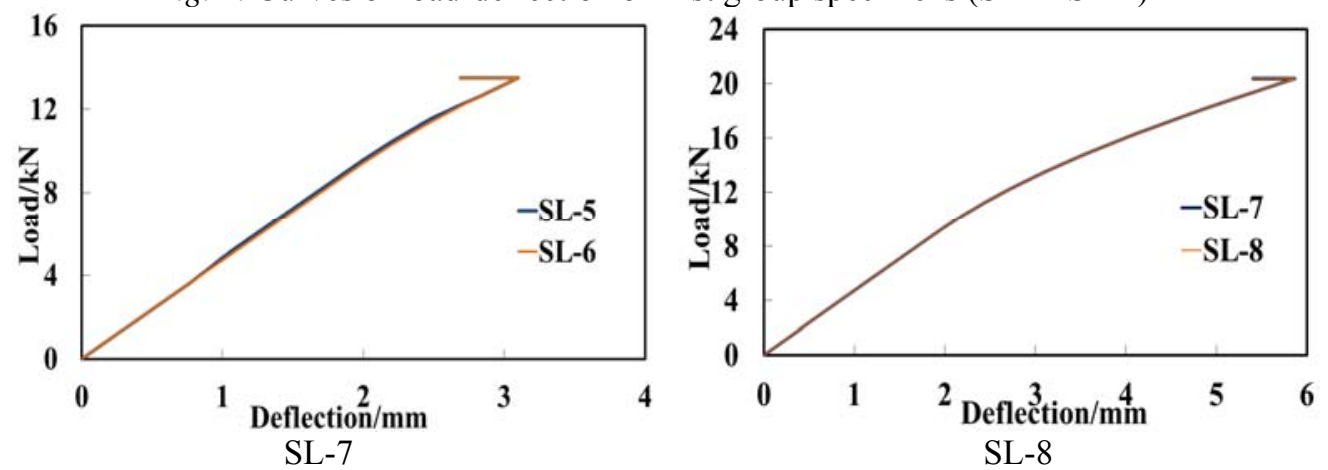

Fig. 5. Curves of load-deflection of second group specimens (SL-5 SL-8)

On the basis of the numerical data, the following conclusions can be drawn:

1. As shown in Fig. 4, all the four smart beams in first group exhibit good capacity of deflection recovery. When eccentricity of the actuator increased from $h / 4$ to $3 \mathrm{~h} / 8$, the recovery ratio of deflection increased by $2.12 \%$ and $3.61 \%$. Meanwhile, when crack width increased from $0.1 \mathrm{~mm}$ to $0.2 \mathrm{~mm}$, the recovery ratio of deflection decreased by $5.03 \%$ and $6.52 \%$. The results indicate that the piezoelectric actuator have greater active control effect when they are located further from the neutral axis of the beam section. Moreover, it can be found that the initial damage of piezoelectric smart concrete beams has an impact on the active control effects. The recovery ratio of deflection is smaller after actuating when the initial damage of piezoelectric smart concrete beam is more severe. 
2. As shown in Fig. 5, good capacity of deflection recovery can also be observed in the second group specimens. When eccentricity of the actuator increased from $\mathrm{h} / 4$ to $3 \mathrm{~h} / 8$, the recovery ratio of deflection increased by $1.79 \%$ and $2.24 \%$. When crack control width increased from $0.1 \mathrm{~mm}$ to $0.2 \mathrm{~mm}$, the recovery ratio of deflection decreased by $2.78 \%$ and $4.99 \%$. Similar conclusions can be drawn from the comparison of the results.

\section{Conclusions}

1. A nonlinear analysis program for the analysis of piezoelectric smart concrete beams under active deflection control is presented. The material nonlinearity, geometric nonlinearity as well as the local contact effects of cracked section are taken into account in the program.

2. Active control tests have been conducted and the results validate that the proposed program is able to perform a good prediction of the active deflection control of piezoelectric smart concrete beams under combined static loading and actuating force.

3. A parametric study has been undertaken and the effects of eccentricity of the actuator and the initial damage of concrete beam on the active deflection control of smart concrete beams with piezoelectric actuators have been studied. It is found that piezoelectric actuators have greater effect when the eccentricity is larger and the ratio of deflection recovery is smaller after actuating when the initial damage of piezoelectric smart concrete beam is more severe.

Besides, the research work of this paper can provide an analytical approach for the future studies of smart structures using piezoelectric actuators in civil engineering.

\section{Acknowledgements}

The authors gratefully acknowledge the financial support of the Scientific Research Project by Shanghai Science and Technology Committee of China (No. 14DZ1208302).

\section{References}

[1] H. Ren and H.Q. Fan: Sensor. Actuat. A-Phys Vol. 128 (2006), p.132-139

[2] L.S. Jang and K.C. Kuo: Sensors Vol. 7 (2007), p. 493-507

[3] I. Chopra: AIAA. J Vol. 40 (2002), p. 2145-2187

[4] Zheng K, Dong X H and Tian X: Mater. Res. Innov Vol. 18 (2014), p. 132-135

[5] G. Song, V. Sethi and H.N. Li: Eng. Struct Vol. 28 (2006), p. 1513-1524

[6] P. Warnitchai, Y. Fujino and B.M. Pacheco: J. Appl. Mechanics Vol.60 (1993), p. 948-953.

[7] Y. Fujino and T. Susumpow: Smart. Mater. Struct Vol.4 (1995), p. A41.

[8] F.R. Rofooei and A. Nikkhoo: Int. J. Solids Struct Vol. 46 (2009), p. 2429-2443

[9] A. Nikkhoo: Mech. Syst. Signal. Pr Vol. 45 (2014), p. 513-530

[10] T. Kamada, T. Fujita, T. Hatayama, T. Arikabe, N. Murai, S. Aizawa and K. Tohyama: Smart. Mater. Struct Vol.6 (1997), p. 448-456

[11] T. Kamada, T. Fujita, T. Hatayama, T. Arikabe, N. Murai, S. Aizawa and K. Tohyama: Smart. Mater. Struct Vol. 7 (1998), p. 479-488

[12] F. Yang: 'Fundamental theories and loading performance of piezoelectric smart beams', Master thesis, Tongji University, Shanghai, China, 2005, 27-45. [In Chinese] 